\author{
K. Hormat, H. Tahiri, N. Guennoun \& A. Gounssa
}

\title{
Étude caryologique d'un taxon endémique du Maroc appartenant au genre Vicia (Leguminosae)
}

\begin{abstract}
Hormat, K. Tahiri, H., Guennoun, N. \& Gounssa, A. 2019: Étude caryologique d'un taxon endémique du Maroc appartenant au genre Vicia (Leguminosae) [In Kamari, G., Blanché, C. \& Siljak-Yakovlev, S. (eds), Mediterranean plant karyological data - 29]. - Fl. Medit. 29: 329-333. http://dx.doi.org/10.7320/FlMedit29.329

A systematic revision of two taxa belonging to the genus Vicia L. section Cracca S.F. Gray, V. benghalensis L. and $V$. villosa Roth, led us to propose, in a previous contribution, a new combination and a new status for an endemic taxon of Morocco: $V$. benghalensis subsp. heterocalyx var. simulans (Maire) Hormat. This taxon, as well as $V$. benghalensis var. benghalensis show a $2 n=14$ chromosome number, whereas $V$. benghalensis subsp. heterocalyx var. heterocalyx gives $2 n=12$.

Keywords: Vicia, Fabaceae, Karyosystematics, Moroccan flora.
\end{abstract}

\section{Introduction}

Vicia benghalensis subsp. heterocalyx var. simulans (Maire) Hormat, taxon endémique marocain de la région de Mehdia, a été décrit pour la première fois par René Maire (1937) et a été rattaché à Vicia villosa Roth en raison de son fruit totalement glabre. Raynaud (1976), dans une révision taxonomique de la section Cracca, a gardé ce taxon au sein de l'espèce $V$. villosa Roth. Lors de notre étude de la section (Hormat 1985, 2004) nous avons remarqué, qu'en plus de son fruit glabre, ce taxon diffère des taxons infra-spécifiques de $V$. villosa par la couleur de la corolle, ce qui nous a amené à faire une étude caryologique qui pourrait nous fournir des renseignements nouveaux pour situer ce taxon au sein de la section Cracca.

\section{Matériel et méthodes}

Nous avons récolté des échantillons fleuris et surtout fructifiés de $V$. benghalensis subsp. heterocalyx var. simulans à Mehdia, seul endroit où il semble exister avec $V$. benghalensis subsp. heterocalyx var. heterocalyx, que nous avons aussi récolté pour comparaison. Pour les besoins de comparaison toujours, nous avons également récolté $V$. villosa subsp. villosa à Tétouan, qui semble exister tout à fait au nord du Maroc et d'Algérie et $V$. benghalensis var. benghalensis à Tanger. Les échantillons récoltés sont déposés à l'herbier de Montpellier (MPU).

L'étude caryologique, à partir des racines obtenues par la germination de graines récoltées, a nécessité deux méthodes de colorations: la méthode au carmin acétique et la méthode à l'or- 
céine acétique pour les plantes qui n'ont pas montré une bonne coloration de chromosomes avec la technique au carmin acétique. A partir des plaques métaphasiques (10 par population) nous avons établi le caryotype et l'idiogramme correspondant, suivis de la formule chromosomique. Le type chromosomique est déterminé par la position du centromère d'après la nomenclature de Levan \& al. (1964). Pour préciser la dimension des chromosomes nous avons établi l'échelle des valeurs reprise de Verlaque (1983) et de Xena De Enrech (1987).

\section{Résultats et discussion}

Au cours de nos observations nous avons pu mettre en évidence deux types de chromosomes : les chromosomes A, ordinaires, qui se présentent sous forme de bâtonnets, de longueur variant de 2 à $7 \mu \mathrm{m}$, et de nombre variant de $2 n=12$ à $2 n=14$; et les chromosomes $\mathrm{B}$, ou surnuméraires, ou accessoires, qui se présentent sous forme de très petits bâtonnets, souvent métacentriques, et dont le nombre est variable même parfois parmi les cellules d'un même individu. Il semble que ces chromosomes B proviennent des chromosomes A par l'intermédiaire de translocations, délétions ou inversions (Fernandes 1949).

1972. Vicia villosa Roth subsp. villosa $-2 n=14$.

Ma: Rif : $6 \mathrm{Km}$ au sud-est de Tétouan, entre Tanger et Tétouan, $30 \mathrm{~m}, 35^{\circ} 45^{\prime} / 5^{\circ} 18^{\prime}$. MPU - HERBIER HORMAT ${ }^{\circ} 157$.

La méthode à l'orcéine a révélé un nombre chromosomique de $2 n=14$ qui est en accord avec les rapports des auteurs qui ont travaillé sur V. villosa s.s., notamment Seen (1938), Baksay (1954), Cincura (1962) et Raina \& Rees (1983), avec toujours la présence de deux chromosomes B, toutefois Baksay a noté la présence de satellites sur une paire de chromosomes. Le caryotype est composé d'une paire de chromosomes moyens, de 4,5 $\mu \mathrm{m}$ environ, à centromère subterminal, 3 paires de chromosomes moyens, de $4 \mu \mathrm{m}$ environ, à centromère submédian, 2 paires de chromosomes moyens, de 3,5 $\mu \mathrm{m}$ environ, à centromère dans la région médiane, une paire de chromosomes très courts, de $2 \mu \mathrm{m}$ environ, à centromère submédian. Ce caryotype est représenté par la formule chromosomique suivante: 6 $\mathrm{M}(1 \mathrm{st}+3 \mathrm{sm}+2 \mathrm{~m})+1 \mathrm{TC}(\mathrm{sm})$.

1973. Vicia benghalensis L. var. benghalensis $-2 n=14$.

Ma: Rif : $30 \mathrm{Km}$ au sud de Tanger, entre Asila et Tanger, dans la forêt diplomatique, 30 $\mathrm{m}, 35^{\circ} 38^{\prime} / 5^{\circ} 57^{\prime}$. HERBIER HORMAT n ${ }^{\circ} 149$.

Selon la méthode à l'orcéine nous avons obtenu des plaques métaphasiques qui montrent $2 n=14$ chromosomes. Le nombre chromosomique de base $\mathrm{x}=7$ est en accord avec celui proposé par des auteurs qui ont travaillé sur ce taxon, notamment Senn (1938), Rajan (1952) et Raina \& Rees (1983). Le caryotype est composé d'une paire de chromosomes 
très longs (environ $7 \mu \mathrm{m}$ ) à centromère submédian, d'une paire de chromosomes longs (environ $6 \mu \mathrm{m}$ ) à centromère submédian, d'une paire de chromosomes longs (environ 5,5 $\mu \mathrm{m})$ à centromère submédian, d'une paire de chromosomes longs (environ $5 \mu \mathrm{m}$ ) à centromère submédian, d'une paire de chromosomes moyens (environ 4,5 $\mu \mathrm{m}$ ) à centromère dans la région médiane, d'une paire de chromosomes moyens (environ $4 \mu \mathrm{m}$ ) à centromère dans la région médiane, et d'une paire de chromosomes courts (environ $3 \mu \mathrm{m}$ ) à centromère médian. Ce caryotype est représenté par la formule chromosomique suivante: 1 TL (sm) $+3 \mathrm{~L}(\mathrm{sm})+2 \mathrm{M}(\mathrm{m})+1 \mathrm{C}(\mathrm{m})$.

On note une différence majeure avec $V$. villosa par les chromosomes qui sont beaucoup plus grands. Ils se rapprochent par la taille et par l'absence de chromosomes subtélocentriques à ceux de $V$. villosa subsp. simulans.

1974. Vicia benghalensis subsp. heterocalyx var. simulans (Maire) Hormat $-2 n=14$.

Ma: Région de Kenitra : Mehdia, tout le long de la Merja de Sidi Bou-Ghaba, $60 \mathrm{~m}, 34^{\circ}$ $14^{\prime} / 6^{\circ} 41^{\prime}$. MPU - HERBIER HORMAT n ${ }^{\circ} 160$.

La méthode au carmin acétique a révélé un nombre diploïde de $2 n=14$ avec présence de chromosomes surnuméraires dont le nombre varie entre 2 et 3 chromosomes B. Ce nombre chromosomique est récent, il n'a jamais fait l'objet d'une recherche caryologique, il se trouve pour la première fois chez ce taxon endémique du Maroc. Le caryotype est composé de 2 paires de chromosomes longs de $5 \mu \mathrm{m}$ environ, à centromère submédian, 2 paires de chromosomes moyens de $4 \mu \mathrm{m}$ environ, à centromère submédian, 2 paires de chromosomes moyens de $3,5 \mu \mathrm{m}$ environ, à centromère submédian ( 1 paire) dans la région médiane (1 paire), 1 paire de chromosomes courtsde $3 \mu \mathrm{m}$ environ, à centromère submédian. La formule chromosomique est la suivante: $2 \mathrm{~L}(\mathrm{sm})+4 \mathrm{M}(3 \mathrm{sm}+1 \mathrm{~m})+1 \mathrm{C}(\mathrm{sm})$.

On remarque que les chromosomes sont un peu plus grands que ceux de $V$. villosa, et qu'il y a absence de chromosomes subtélocentriques.

1975. Vicia benghalensis subsp. heterocalyx var. heterocalyx (Maire-Weiller) Hormat $2 n=12$.

Ma: Région de Kenitra : Mehdia, tout le long de la Merja de Sidi Bou-Ghaba, 60 m, 34 $14^{\prime} / 6^{\circ}$ 41. MPU - HERBIER HORMAT n 150.

Selon la méthode au carmin acétique, dans les métaphases somatiques nous avons dénombré $2 n=12$ chromosomes, et nous avons noté la présence de chromosomes surnuméraires dont le nombre est variable ( 5 , 6 à 8 chromosomes $\mathrm{B})$. Le nombre chromosomique de base $\mathrm{x}=6$ est récent et trouvé pour la première fois chez ce taxon, toutefois il a été également indiqué chez $V$. benghalensis var. benghalensis par Srivastava (1963). Les chromosomes sont en général moins grands que ceux de $V$. benghalensis var. benghalensis. Le caryotype correspondant aux idiogrammes montre: une paire de chromosomes longs d'environ $6 \mu \mathrm{m}$, à centromère submédian, 2 paires de chromosomes longs d'environ $5 \mu \mathrm{m}$, 
à centromère submédian, 2 paires de chromosomes moyens ( $4,5 \mu \mathrm{m}$ environ) à centromère submédian, et une paire de chromosomes moyens de $4 \mu \mathrm{m}$ à centromère submédian. La formule chromosomique est la suivante: $3 \mathrm{~L}(\mathrm{sm})+3 \mathrm{M}(\mathrm{sm})$.

On remarque une ressemblance entre les caryotypes de $V$. benghalensis subsp. heterocalyx var. heterocalyx et de $V$. benghalensis var. benghalensis, sauf pour les chromosomes de la première paire et ceux de la dernière paire. On peut se demander si c'est la première paire ou si c'est la septième paire chez $V$. benghalensis s.s. qui s'est fragmentée pour donner les chromosomes B chez $V$. benghalensis subsp. heterocalyx var. heterocalyx. Les chromosomes s'approchent beaucoup de ceux de Vicia benghalensis subsp. heterocalyx var. simulans (Maire) Hormat par la taille et par l'absence de chromosomes subtélocentriques.

\section{Conclusion}

L'étude caryologique réalisée dans ce groupe met en évidence l'existence de deux nombres chromosomiques $2 n=12$ et $2 n=14$, avec l'absence ou la présence de chromosomes surnuméraires.

On peut penser que le nombre de base $\mathrm{x}=6$ provient d'une diminution du nombre de base $x=7$ par le phénomène de la dysploidie descendante (Senn 1938; Goldblatt 1981; Polhill \& al. 1981). Il faut noter aussi qu'il y a chez tous les taxons étudiés l'absence de chromosomes télocentriques $(\mathrm{t})$ et de chromosomes acrocentriques $(\mathrm{T})$.

L'examen des caryotypes entraine certaines constatations: $V$. benghalensis s.s. se distingue de $V$. villosa s.s. par la forme de leurs chromosomes; en effet les chromosomes les plus longs à centromère submédian ou médiane existent chez $V$. benghalensis. Chez $V$. villosa les chromosomes sont petits et la première paire de chromosomes moyens est toujours à centromère subterminal comme l'estime Roti-Michelozi (1986). Vicia benghalensis subsp. heterocalyx var. simulans a des chromosomes un peu plus grands que ceux de $V$. villosa et l'absence de chromosomes subtélocentriques (caryotype symétrique) le rapproche de $V$. benghalensis.

Vicia benghalensis subsp. heterocalyx var. heterocalyx possède des chromosomes relativement grands analogues à ceux de $V$. benghalensis et jamais de chromosomes subtélocentriques comme $V$. benghalensis subsp. heterocalyx var. simulans. La perte de deux chromosomes de $2 n=14$ à $2 n=12$ s'explique par des translocations inégales par lesquelles un chromosome devient si petit qu'il finit par se perdre après d'être fragmenté pour donner naissance à des chromosomes B (Favarger 1962; Hollings \& Stace 1974; Bandel 1974).

En conclusion, $V$. benghalensis subsp. heterocalyx var. simulans, rattachée à l'espèce $V$. villosa par Maire (1937) en se basant sur le seul critère du fruit glabre, c'est bien qu'il soit exclue et mieux placée au sein de l'espèce $V$. benghalensis, comme indiqué in Hormat (2004), aussi par la présence de grands chromosomes et par l'absence de chromosomes subtélocentriques.

\section{Références}

Baksay, L. 1954: Chromosomen studien an der ungarischen Vicia L., Arten. - Ann. His. Nat. Musei Nat. Hung. 5: 139-148. 
Bandel, G. 1974: Chromosome numbers and evolution in the Leguminosae. - Caryologia 27(1): 17-32. https://doi.org/10.1080/00087114.1974.10796558

Cincura, F. 1962: Poznamky K cytologii druhov rodu Vicia L. zo slovenskychna lezisk. - Acta Fac. Rerum. Nat. Univ. Comenianae Bot. 7(6-7): 349-371.

Favarger, C. 1962: L'évolution parallèle du caryotype. - Rev. Cytol. Biol. Vég. 25(3-4): 277-286. https://doi.org/10.1080/00378941.1962.10838105

Fernandes, A. 1949 : Sur la caryosystématique de la section Ganimedes (Salisb.) Schult.F. du genre Narcissus L. - Bull. Soc. Brot. 23(1): 177-219.

Goldblatt, P. 1981: Cytology and the phylogeny of Leguminosae. - Pp. 427-463 in: Polhill, R. M. \& Raven, P. H. (eds), Advances in legume systematic. - London.

Hollings, E. \& Stace, C. A. 1974: Karyotype variation and evolution in Vicia sativa. - New Phytol. 73: 195-208. https://doi.org/10.1111/j.1469-8137.1974.tb04619.x

Hormat, K. 1985: Le genre Vicia L. section Cracca (L.) S. F. Gray Au Maroc - Approche systématique. - Montpellier.

- 2004: Données nouvelles sur Vicia villosa Roth subsp. simulans Maire (Leguminosae) taxon endémique du Maroc. - Lazaroa 25: 143-151.

Levan, A., Fredga, K. \& Sandeberg, A. A. 1964: Nomenclature for centromeric position on chromosomes. - Hereditas 52(2): 201-220. https://doi.org/10.1111/j.1601-5223.1964.tb01953.x

Maire, R. 1928: Contribution à l'étude de la Flore de l'Afrique du Nord. - Bull. Soc. Hist. Nat. Afr. Nord 2(17): 44, 2(18): 288.

- 1937: Contribution à l'étude de la Flore de l'Afrique du Nord. - Bull. Soc. Hist. Nat. Afr. Nord 28(25): 353.

Polhill, R. M., Raven, P. H. \& Stirton, C. H. 1981: Evolution and systematics of the Leguminosae. Pp. 1-26 in: Polhill, R. M. \& Raven, P. H. (eds), Advences in legume ststematics. - London.

Raina, S. N., \& Rees, H. 1983: DNA variation between and within chromosome complement of Vicia species. - Heredity 51: 335-346. https://doi.org/10.1038/hdy.1983.38

Rajan, S. S. 1952: A note on the chromosome numbers of some plants. - Indian J. Genet. Pl. Br. 12(2): 93.

Raynaud, C. 1976: Monographie et iconographie du genre Vicia au Maroc. - Bull. Inst. Sci. 1: 147-172.

Roti-Michellozzi, G. 1986: Biosystematic studies on the V. villosa complex in Europe. - Condollea 41(2): 399-411.

Seen, H. 1938: Chromosome number relationships in the Leguminosae. - Bibliogr. Genet. 12: 175-336.

Srivastava, L. N. 1963: Cytogenetical studies in certain species of Vicia. - Cytologia 28: 154-161.

Verlaque, R. 1983: Etude biosystématique et phylogénétique des Dipsacacées. - Thèse de doctorat, Université De Provence - Aix Marseille I - Centre St Charles.

Xena De Enrech, N. 1987: Recherche biosystématique sur le genre Fedia (Valerianacées). - Thèse de doctorat, Université de Montpellier.

Adresse des auteurs:

Kamal Hormat, Hikmat Tahiri, Naima Guennoun \& Abdelkarim Gounssa, Centre de Biotechnologies Végétale et Microbienne, Biodiversité et Environnement (BioBio), Equipe de Botanique et Valorisation des Ressources Végétales et Fongiques (BOVAREF), Département de Biologie, Faculté des Science, Mohammed V University in Rabat. 4 Avenue Ibn Battouta, B.P. 1014 RP, Rabat, Maroc. E-mails: khormat@fsr.ac.ma; tahiri47@gmail.com; naimaguennoun@gmail.com; abdelkarimgounssa@gmail.com 